\title{
Ultraviolet-activated long-lived room-temperature phosphorescence from small organic molecule-doped polymer systems
}

\author{
Zhonghao Wang ${ }^{1 \dagger}$, Yan Zheng ${ }^{1 \dagger}$, Yan $\mathrm{Su}^{1 \dagger}$, Liang Gao ${ }^{1}$, Yinyin $\mathrm{Zhu}^{1}$, Jie Xia ${ }^{1}$, Yongfeng Zhang ${ }^{1}$, \\ Chang Wang ${ }^{1}$, Xian Zheng ${ }^{1}$, Yanli Zhao ${ }^{2 *}$, Chaolong Yang ${ }^{1,2^{*}}$ and Youbing $\mathrm{Li}^{1}$
}

\begin{abstract}
Long-lived organic room-temperature phosphorescent (RTP) materials have attracted widespread attention because of their fantastic properties and application prospects. The current methods for developing RTP materials are mainly based on the synthesis of new chromophore molecules and crystallization engineering. However, there are great challenges in the preparation of new chromophore molecules and the use of crystalline materials. Herein, dynamic stimulus-responsive long-lived RTP systems with various emission colors are realized by doping organic chromophore molecules into polymer matrix prepared from vinyl acetate and acrylic acid. Through UV light irradiation, the growth process of long-lived RTP phenomena can be observed for up to $10 \mathrm{~s}$. In particular, the phosphorescence intensity, lifetime, afterglow brightness, and quantum yield of one representative film (P2-M2) increase by 155, 262, 414, and 8 times after the irradiation, respectively. The unique photophysical phenomena are ascribed to the oxygen consumption characteristics of the polymer matrix under UV irradiation. Meanwhile, the information storage devices are prepared with these RTP systems. This work provides a strategy for achieving small organic molecule-doped polymer RTP systems that are easy to prepare, low-cost, and widely adaptable.
\end{abstract}

Keywords: information storage, luminescent materials, phosphorescence growth, responsive polymers, room temperature phosphorescence

\section{INTRODUCTION}

Stimulus-responsive luminescent materials are a type of smart materials, and their luminous performance can be adjusted by applying external stimuli such as light, force and temperature [1-4]. Because of their unique photophysical properties, they have attracted widespread attention, showing an important application prospect in the fields of optoelectronic devices, imaging, sensing, and information encryption/decryption $[5,6]$. Organic long-lived room-temperature phosphorescence (RTP) materials can continuously emit light for a period of time after the excitation source is removed [7-11]. In the past, the organic
RTP phenomenon was usually found at low temperatures (such as $77 \mathrm{~K}$ ). Because the nonradiative transition of organic molecules is strong, the triplet excitons could be easily quenched by oxygen molecules in the atmosphere [12]. To address this issue, some effective methods have been developed to prepare organic RTP materials by promoting intersystem crossing (ISC) and suppressing nonradiative transitions, which include halogen bonding $[13,14]$, crystallization engineering $[15,16]$ and others [17-23].

At present, the main methods for developing RTP materials are to synthesize new chromophore molecules and to carry out the crystallization engineering, since the chromophore crystals can effectively compress the molecular vibration and isolate oxygen [24]. However, it is still difficult to develop new chromophore molecules with excellent RTP performance, and the cost associated with the design and preparation is usually high. While these RTP materials are expected to be used in the forms of films or nanoparticles, their crystalline nature results in difficulties in processability, flexibility, and transparency for applications $[25,26]$. Considering the intriguing features of polymer films, such as flexibility, lightweight, transparency, stretchability, and low cost, it is necessary to investigate longlived polymer RTP systems at ambient conditions. Some efforts have been devoted to developing polymers with long-lived RTP by embedding chromophores into the polymer matrix [2,21], or bonding chromophores to the backbone of polymers [27,28]. Nevertheless, the synthesis of new chromophores and long-lived polymer RTP materials is still complicated, time-consuming, and expensive. Furthermore, most of the RTP materials are static, and the long-lived polymer RTP systems with a dynamic stimulus-responsive behavior have been rarely reported [29-35]. Compared with static RTP materials, dynamic stimulusresponsive RTP systems have more application prospects in the fields of sensing, as well as information anticounterfeiting and encryption. Therefore, it is highly desirable to discover a general, efficient, and low-cost method for the development of stimulusresponsive polymer RTP materials with excellent properties and versatile applicability.

Under UV light irradiation, the triplet exciton of chromophore molecules can transfer energy to triplet oxygen to gen-

\footnotetext{
${ }^{1}$ School of Materials Science and Engineering, Chongqing University of Technology, Chongqing 400054, China

${ }^{2}$ Division of Chemistry and Biological Chemistry, School of Physical and Mathematical Sciences, Nanyang Technological University, Singapore 637371, Singapore

$\dagger$ These authors contributed equally to this work.

* Corresponding authors (emails: zhaoyanli@ntu.edu.sg (Zhao Y); yclzjun@163.com (Yang C))
} 
erate singlet oxygen, resulting in the quenching of the triplet exciton [36]. In the meanwhile, singlet oxygen can be consumed by oxidizing the polymer matrix [37]. Recently, some typical radiation-dependent RTP materials have been developed using polymethyl methacrylate (PMMA) as the oxygen consumption matrix [38-41]. However, there are still some issues that need to be solved. For example, the influences of molecular structures of polymers and chromophores on the photoresponsive behavior of the resulting materials are still unclear, the performance of longlived RTP using PMMA as the oxygen-consuming matrix requires further improvements, and there is a need to develop environmentally friendly polymer matrices. Therefore, it is significant to explore novel polymer matrices for achieving excellent radiation-dependent RTP performance through the oxygen consumption strategy. Herein, we report a series of irradiationdependent RTP systems by doping common chromophore molecules into newly developed oxygen-consuming polymer matrices. The growth process of the long-lived RTP phenomenon from scratch can be obviously observed under UV $254 \mathrm{~nm}$ irradiation for up to $10 \mathrm{~s}$. After the irradiation, the phosphorescence intensity, lifetime, afterglow brightness, and quantum yield of a representative doped polymer film increase by 155 , 262,414 , and 8 times, respectively.

\section{EXPERIMENTAL SECTION}

Synthesis of P2 (VAc:AA = 4:1): vinyl acetate (VAc, $10 \mathrm{~mL}$ ), acrylic acid (AA, $1.85 \mathrm{~mL}$ ), 2,2-azobisisobutyronitrile (AIBN, recrystallization from methanol, $60 \mathrm{mg}$ ), 4-(benzenecarbonothioylsulfanyl)-4-cyanopentanoic acid (reversible additionfragmentation chain transfer (RAFT) polymerization reagent, $30 \mathrm{mg})$ and methanol $(20 \mathrm{~mL})$ were added into a Schlenk tube. After repeated evacuation and ventilation with argon, the reaction mixture was treated at $90^{\circ} \mathrm{C}$ for $24 \mathrm{~h}$ to obtain a viscous liquid, which was poured into a large amount of deionized water for reprecipitation. After drying at $65^{\circ} \mathrm{C}$ in a vacuum environment for $24 \mathrm{~h}$, the final product was obtained. ${ }^{1} \mathrm{H}$ nuclear magnetic resonance (NMR) $\left(400 \mathrm{~Hz}, \mathrm{DMSO}-\mathrm{d}_{6}\right): \delta 12.26(\mathrm{~s}, 1 \mathrm{H})$, 4.83-4.74 $(\mathrm{m}, 1 \mathrm{H})$. Fourier transform infrared spectroscopy (FTIR): $3514 \mathrm{~cm}^{-1} . M_{\mathrm{w}}=22,479$ Da. $M_{\mathrm{n}}=12,684$ Da. Polydispersity index $(\mathrm{PDI})=1.77$.

Synthesis of PVAc: $10 \mathrm{~mL}$ of VAc, $10 \mathrm{~mL}$ of methanol, and no $\mathrm{AA}$ addition were applied. The rest of the reagent dosage and synthesis steps are the same as the synthesis of P2. ${ }^{1} \mathrm{H}$ NMR $\left(400 \mathrm{~Hz}, \mathrm{DMSO}-\mathrm{d}_{6}\right): \delta 4.83-4.74(\mathrm{~m}, 1 \mathrm{H}) \cdot M_{\mathrm{w}}=27,158$ Da. $M_{\mathrm{n}}=$ $9701 \mathrm{Da}$. PDI $=2.80$.

Synthesis of P1 (8:1): $10 \mathrm{~mL}$ of VAc, $0.93 \mathrm{~mL}$ of AA, and $10 \mathrm{~mL}$ of methanol were applied. The rest of the reagent dosage and synthesis steps are the same as the synthesis of P2. ${ }^{1} \mathrm{H}$ NMR $\left(400 \mathrm{~Hz}, \mathrm{DMSO}-\mathrm{d}_{6}\right): \delta 12.26(\mathrm{~s}, 1 \mathrm{H}), 4.83-4.74(\mathrm{~m}, 1 \mathrm{H})$. FTIR: $3514 \mathrm{~cm}^{-1} \cdot M_{\mathrm{w}}=30,828$ Da. $M_{\mathrm{n}}=13,719 \mathrm{Da}$. PDI $=2.25$.

Synthesis of P3 (2:1): $10 \mathrm{~mL}$ of VAc, $3.7 \mathrm{~mL}$ of AA, and $25 \mathrm{~mL}$ of methanol were applied. The rest of the reagent dosage and synthesis steps are the same as the synthesis of $\mathrm{P} 2 .{ }^{1} \mathrm{H}$ NMR $\left(400 \mathrm{~Hz}, \mathrm{DMSO}-\mathrm{d}_{6}\right): \delta 12.34(\mathrm{~s}, 1 \mathrm{H}), 4.80-4.76(\mathrm{t}, 1 \mathrm{H})$. FTIR: $3514 \mathrm{~cm}^{-1} . M_{\mathrm{w}}=34,510 \mathrm{Da} . M_{\mathrm{n}}=17,690 \mathrm{Da}$. PDI $=1.95$.

Synthesis of P4 (1:1): $10 \mathrm{~mL}$ of VAc, $7.4 \mathrm{~mL}$ of AA, and $35 \mathrm{~mL}$ of methanol were applied. The rest of the reagent dosage and synthesis steps are the same as the synthesis of P2. ${ }^{1} \mathrm{H}$ NMR $\left(400 \mathrm{~Hz}, \mathrm{DMSO}-\mathrm{d}_{6}\right): \delta 12.27$ (s, 1H), 4.81 (s, 1H). FTIR: $3514 \mathrm{~cm}^{-1} \cdot M_{\mathrm{w}}=26,776$ Da. $M_{\mathrm{n}}=15,063 \mathrm{Da}$. PDI $=1.78$.

Synthesis of P5 (1:2): $5 \mathrm{~mL}$ of VAc, $7.4 \mathrm{~mL}$ of AA, $25 \mathrm{~mL}$ of methanol, $30 \mathrm{mg}$ of $\mathrm{AIBN}$, and $15 \mathrm{mg}$ of RAFT reagent were applied. The solvent used in the final reprecipitation step was ethyl acetate. The rest of the reagent dosage and synthesis steps are the same as the synthesis of P2. ${ }^{1} \mathrm{H}$ NMR $(400 \mathrm{~Hz}$, DMSO$\left.\mathrm{d}_{6}\right): \delta 12.26-12.25(\mathrm{~d}, 1 \mathrm{H}), 4.80(\mathrm{~s}, 1 \mathrm{H})$. FTIR: $3514 \mathrm{~cm}^{-1} . M_{\mathrm{w}}=$ 23,882 Da. $M_{\mathrm{n}}=17,433$ Da. PDI $=1.37$.

Synthesis of P6 (1:4): $5 \mathrm{~mL}$ of VAc, $14.8 \mathrm{~mL}$ of AA, and $35 \mathrm{~mL}$ of methanol were applied. The rest of the reagent dosage and synthesis steps are the same as the synthesis of P5. ${ }^{1} \mathrm{H}$ NMR $\left(400 \mathrm{~Hz}, \mathrm{DMSO}_{-} \mathrm{d}_{6}\right): \delta 12.23(\mathrm{~s}, 1 \mathrm{H}), 4.80$ (s, 1H). FTIR: $3514 \mathrm{~cm}^{-1} \cdot M_{\mathrm{w}}=19,144$ Da. $M_{\mathrm{n}}=14,160 \mathrm{Da}$. PDI $=1.35$.

Synthesis of P7 (1:8): $2.5 \mathrm{~mL}$ of VAc, $14.8 \mathrm{~mL}$ of AA, and $35 \mathrm{~mL}$ of methanol were applied. The rest of the reagent dosage and synthesis steps are the same as the synthesis of P5. ${ }^{1} \mathrm{H}$ NMR $\left(400 \mathrm{~Hz}, \mathrm{DMSO}_{-} \mathrm{d}_{6}\right): \delta 12.24(\mathrm{~s}, 1 \mathrm{H}), 4.79(\mathrm{~s}, 1 \mathrm{H})$. FTIR: $3514 \mathrm{~cm}^{-1} \cdot M_{\mathrm{w}}=107,740$ Da. $M_{\mathrm{n}}=9341 \mathrm{Da}$. PDI $=11.53$.

Synthesis of PAA: $7.4 \mathrm{~mL}$ of AA, $20 \mathrm{~mL}$ of methanol, and no VAc addition were applied. The rest of the reagent dosage and synthesis steps are the same as the synthesis of P5. ${ }^{1} \mathrm{H}$ NMR $\left(400 \mathrm{~Hz}\right.$, DMSO-d $\left.\mathrm{d}_{6}\right): \delta 12.34(\mathrm{~s}, 1 \mathrm{H})$. FTIR: $3514 \mathrm{~cm}^{-1} . M_{\mathrm{w}}=$ 43,566 Da. $M_{\mathrm{n}}=6334$ Da. PDI $=6.88$.

Preparation process of the luminescent films: the preparation method of P2-M2 (a simple organic molecule 4,4'-biphenol was defined as M2) film was taken as an example to describe the preparation of luminescent films in detail. Firstly, P2 (300 mg) and M2 (1 mg) were dissolved in methanol $(10 \mathrm{~mL})$ at $70^{\circ} \mathrm{C}$ to form a solution, and then $0.3 \mathrm{~mL}$ of this solution was dropcoated on the quartz with $1 \times 1 \mathrm{~cm}^{2}$ size. Finally, the film was dried at $60^{\circ} \mathrm{C}$ for $2 \mathrm{~h}$ to afford the P2-M2 film.

\section{RESULTS AND DISCUSSION}

The copolymer (defined as P2) based on VAc and AA as starting materials was successfully synthesized by using reversible addition-fragmentation chain transfer polymerization method (Fig. 1a). The feeding ratio of VAc and AA is 4:1 (molar ratio). The ${ }^{1} \mathrm{H}$ NMR spectral analysis shows that the ratio of VAc and AA in the final product is $3.5: 1$, which is close to the feeding ratio. Then, M2 was doped into the polymer matrix to form a high-quality film. In our previous work $[2,42]$, polyvinyl alcohol (PVA) was chosen as the matrix. Its molecular structure contains a large number of hydroxyl groups that can form hydrogen bonding interactions with the doped chromophores to suppress the vibration of the resulting system. In that case, when the formed film is excited by UV light, it shows a bright lightemitting phenomenon, and continues to emit afterglow for a period of time after turning off the UV lamp. Different from our previous work, a new polymer P2 synthesized from VAc and AA was employed as the matrix to trigger the growth of long-lived RTP. Because P2 mainly consists of $-\mathrm{COOCH}_{3}$ group and a low content of $-\mathrm{COOH}$ group, it cannot form strong hydrogen bonding interactions with the doped chromophores, resulting in relatively high molecular vibration in the system. At the same time, the triplet oxygen in the film would further quench the triplet exciton (Fig. 1b). Since the excitation of the triplet state cannot be stabilized (Fig. 1c), the long-lived RTP cannot be achieved when the film is rapidly excited by a UV lamp ( $254 \mathrm{~nm}$, $400 \mu \mathrm{W} \mathrm{cm}{ }^{-2}$ ). When the film is irradiated by the UV lamp for several seconds, it can continuously generate triplet exciton to convert triplet oxygen into excited singlet oxygen, reducing the quenching of triplet exciton. Thus, more obvious phosphorescence can be observed with the naked eye during the irradiation 
$\mathbf{a}$
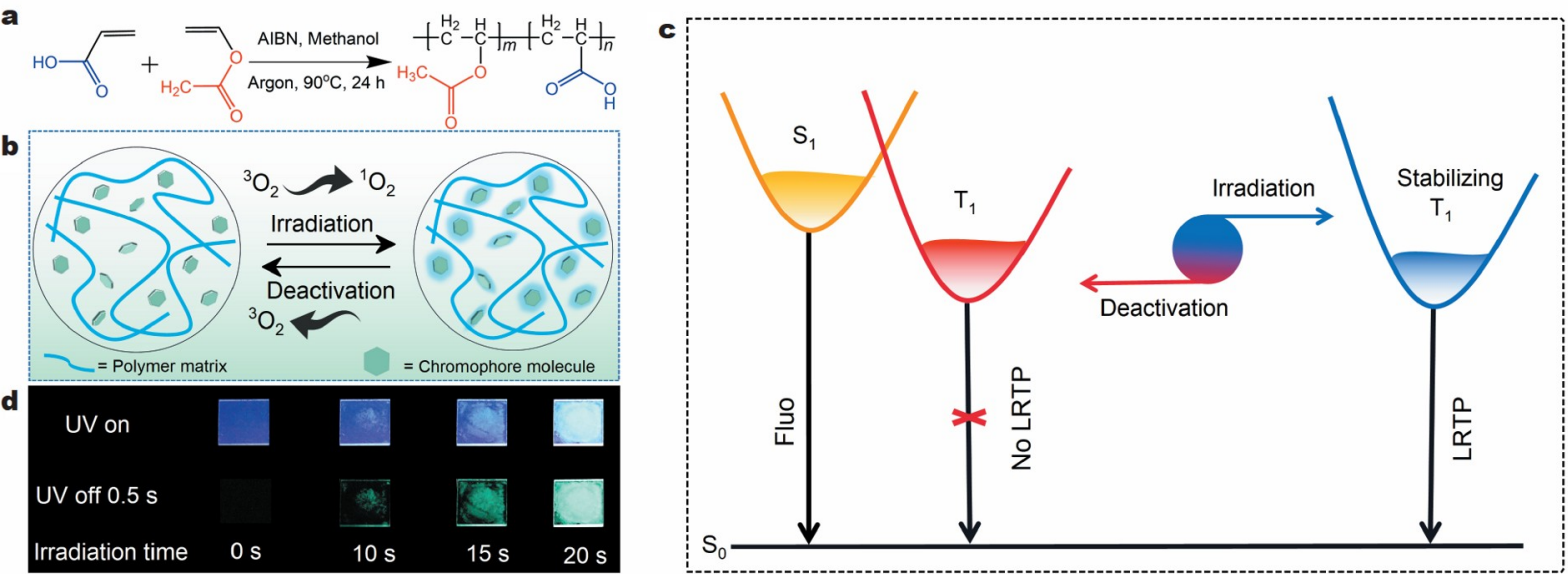

Figure 1 Schematic mechanism and physical images of dynamic stimulus-responsive RTP systems based on doped polymers. (a) Synthetic routes of polymers. (b) Luminescence state of the chromophore molecules in the film before and after the irradiation, along with the cycle of triplet oxygen. (c) Schematic diagram for the energy levels of the doped chromophore molecules before and after the irradiation. Fluo: fluorescence; LRTP: long-lived roomtemperature phosphorescence. (d) Image changes of P2-M2 film during the irradiation.

process (Fig. 1d). The significant RTP phenomenon even under low power UV light excitation indicates that this doped system possesses a sensitive response to UV light stimulation.

The synthetic procedure of the polymer P2 is shown in Fig. S1. P2 was characterized by FTIR spectroscopy (Fig. S2), ${ }^{1} \mathrm{H}$ NMR spectroscopy (Fig. S3) and gel permeation chromatography. Then, P2 was configured into a $30 \mathrm{~g} \mathrm{~L}^{-1}$ methanol solution, and M2 (Fig. S4) was added into the solution at a concentration of $0.1 \mathrm{mg} \mathrm{mL}^{-1}$. The mixture was dropped onto the quartz, and dried at $60^{\circ} \mathrm{C}$ to form a film with a thickness of $30 \mu \mathrm{m}$ (defined as $\mathrm{P} 2-\mathrm{M} 2)$. When the $\mathrm{P} 2-\mathrm{M} 2$ film in the pristine state was rapidly excited with UV light, only very weak phosphorescence at $488 \mathrm{~nm}$ was generated. The phosphorescence lifetime was only $2.7 \mathrm{~ms}$, the phosphorescence quantum yield was $0.98 \%$, and the afterglow brightness was $0.02 \mathrm{mcd} \mathrm{cm}^{-2}$, which cannot be observed by the naked eye or camera. However, when the film was irradiated by the microsecond lamp $\left(40 \mu \mathrm{W} \mathrm{cm}{ }^{-2}\right)$ from the steady-state transient fluorescence spectrometer, the phosphorescence intensity at $488 \mathrm{~nm}$ remarkably increased by over 150 times, and the intensity became stable after the irradiation for $5 \mathrm{~min}$ (Fig. 2a, b and Fig. S5). After $5 \mathrm{~min}$ irradiation, the phosphorescence lifetime increased to $707 \mathrm{~ms}$ (Fig. 2c), the phosphorescence quantum yield enhanced to $8.28 \%$ (Table S1), and the afterglow brightness increased to $8.27 \mathrm{mcd} \mathrm{m}^{-2}$ (Fig. 2d), which are 262,8 , and 414 times higher than that of the pristine state, respectively. Such remarkable improvement under a short irradiation time indicates that the system has an efficient UV light irradiation-responsive behavior.

Impressively, this increasing RTP phenomenon can be observed under UV light even with a low power density $\left(20 \mu \mathrm{W} \mathrm{cm}{ }^{-2}\right.$, Fig. 2e). When using a UV lamp with a power density of $1200 \mu \mathrm{W} \mathrm{cm} \mathrm{cm}^{-2}$, RTP can be generated by only $5 \mathrm{~s}$ of irradiation (Fig. S6). The resolution of the system was measured with a standard USAF 1951 test target (Fig. S7), giving 812 PPI (points per inch). When the film was stored in the atmosphere condition, triplet oxygen would permeate the film and cause the phosphorescence quenching. After being stored for $16 \mathrm{~min}$ and then quickly excited by UV light, the RTP phenomenon disappeared (Fig. 2f). The RTP recovery can be accelerated by heating $\left(90^{\circ} \mathrm{C}\right.$ for $\left.2 \mathrm{~min}\right)$. P2-M2 films with different thicknesses were used to further study this radiation-dependent RTP. When the film thickness reduced to 10 or $20 \mu \mathrm{m}$, the irradiation time required to generate RTP was similar to that of $30-\mu \mathrm{m}$ film (Fig. S8), and the RTP performance after the irradiation was also similar (Figs S9 and S10). However, the inactivation time would be shortened as the thickness decreased. When the film thickness was $10 \mu \mathrm{m}$, it only took $8 \mathrm{~min}$ to completely inactivate it (Fig. $2 \mathrm{f}$ and Fig. S11). When the thickness was $30 \mu \mathrm{m}$, the film can be stored for $16 \mathrm{~min}$, indicating that the decrease in thickness is conducive to the oxygen permeation. After storing the film in a dry environment at room temperature for 10 days, the intensity after the irradiation kept $96 \%$ of the initial time, indicating that the system has a good stability (Fig. S12). The excitation-phosphorescence mapping (Fig. 2g) and transient photoluminescence decay images (Fig. $2 \mathrm{~h}$ ) of the film were also recorded. The results show that the use of 210 to $350 \mathrm{~nm} \mathrm{UV}$ light can effectively trigger the system to produce RTP. Subsequently, a cycle test on this dynamic stimulus-responsive behavior was carried out. After 50 cycles, the phosphorescence intensity of the emission peak at $488 \mathrm{~nm}$ after the irradiation is about $80 \%$ of the initial state (Fig. $2 \mathrm{i}$ ).

In order to further understand the dynamic stimulusresponsive RTP behavior, we screened out 8 other small molecules, and doped them into the P2 matrix to form corresponding films. These small chromophores are 4-phenylbenzoic acid, 3,6diphenyl-9H-carbazole, 4-phenylphenol, 2-hydroxycarbazole, $7 H$-dibenzo[ $c, g]$ carbazole, 3,3'-bicarbazole, methyl vanillate and 1,4,5,8-naphthalenetetracarboxylic dianhydride (defined as M1, M3, M4, M5, M6, M7, M8, and M9, respectively), and their molecular structures are shown in Fig. S4. No RTP was observed for all films in their pristine states when excited by UV light for a short time. Upon the irradiation by UV light for a longer period of time, the increased RTP phenomena from these films were observed. Afterglow color of P2-M5 and P2-M8 films is blue, P2M6 film is yellow, P2-M9 film is red, and the rest is green (Fig. 3a, Fig. S13, and Movies S1 and S2). Corresponding Commission International de l'Eclairage (CIE) coordinates are presented in Fig. S14. Experimental results reveal that different films require different irradiation time and recovery time for the phosphorescence growth (Table S2). Among them, the phos- 

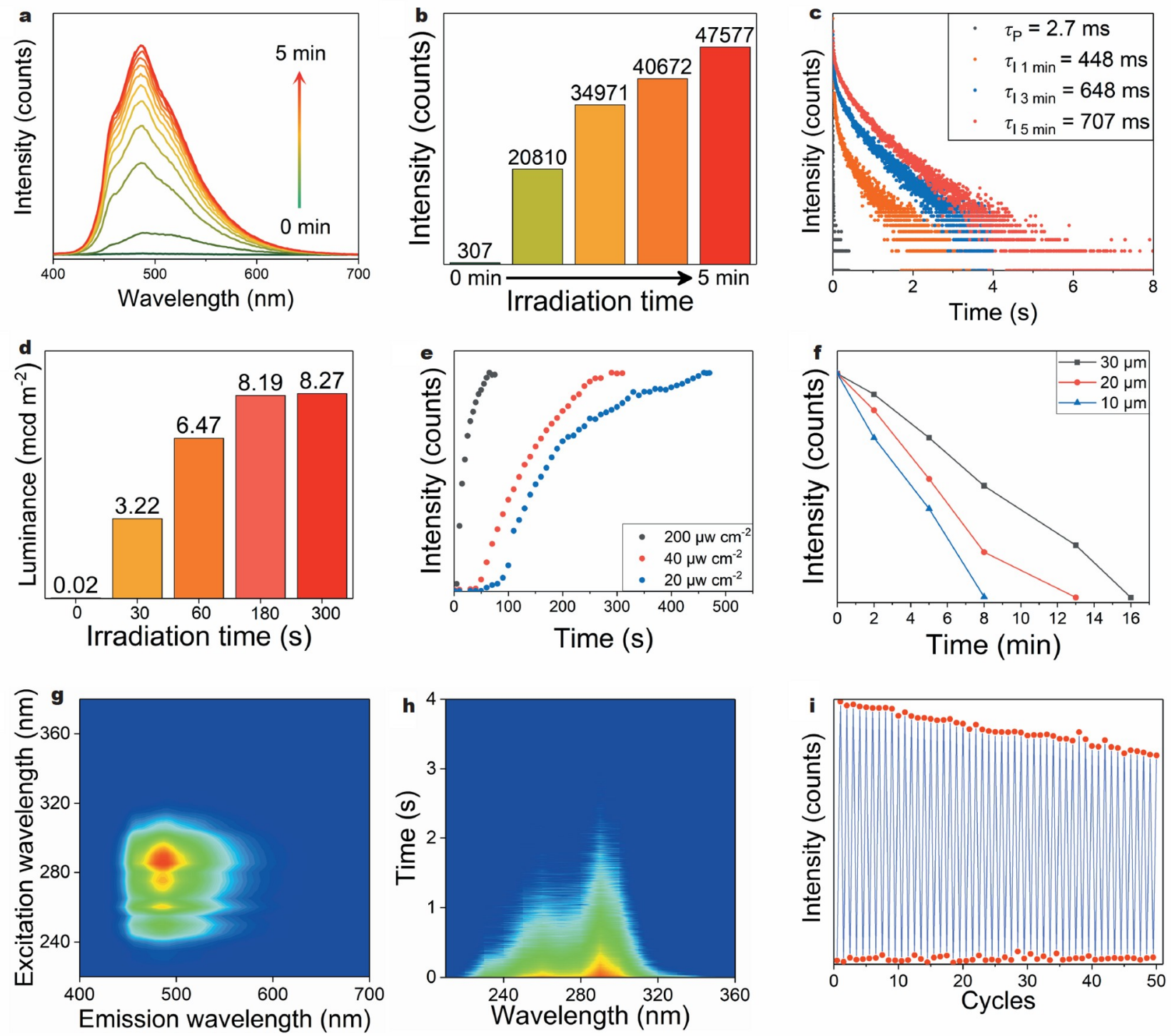

Figure 2 Photophysical properties of the P2-M2 film before and after the irradiation. (a) Phosphorescence spectra of the P2-M2 film upon the irradiation with different irradiation times. The excitation wavelength is $288 \mathrm{~nm}$. (b) Emission intensity changes at $488 \mathrm{~nm}$ under different irradiation times. (c) Phosphorescence lifetime spectra of the emission peak at $488 \mathrm{~nm}$ under different irradiation times. (d) Afterglow brightness of P2-M2 film under different irradiation times. (e) Time required to irradiate the film by UV light under different power densities to reach the RTP saturation. (f) Phosphorescence intensity changes of films with different thicknesses after 5 min of irradiation in the atmosphere conditions. (g) Excitation-phosphorescence mapping of the P2-M2 film. (h) Time-resolved emission spectra of the P2-M2 film upon excitation at $288 \mathrm{~nm}$. (i) Phosphorescence intensity changes of the emission peak at $488 \mathrm{~nm}$ over 50 cycles.

phorescence intensity of the P2-M4 film increases the most after being irradiated, which is 251 times higher than its pristine state (Fig. S15). The phosphorescence lifetime of P2-M7 film extends the most after being irradiated, which is 380 times longer than its pristine state (Fig. 3b and Fig. S16). Due to the presence of residual fluorescence signal, there is a slight difference in the phosphorescence spectra before and after the irradiation. In the initial state, the phosphorescence intensity of the film is very low. After the irradiation, the phosphorescence intensity of the film increases by hundreds of times. Thus, the residual fluorescent signal could be ignored (Fig. S17). The afterglow brightness of all films after the irradiation is shown in Fig. S18, which can last up to 10 s (Figs S19 and S20).

Because of the appearance of RTP, the photoluminescence phenomena of these films were also changed (Fig. S21). The steady-state photoluminescence spectra (Fig. S22) show that the phosphorescence intensity increases with a slight decrease in fluorescence intensity after the irradiation. The fluorescence lifetime after the irradiation is shown in Fig. S23. Meanwhile, the instrument response function (IRF) was also measured (Fig. S24). Their UV absorption spectra, excitation spectra and emission spectra were compared (Fig. S25). Excitation-phosphorescence mapping (Fig. S26) indicates that all films can be irradiated by UV light with a wide wavelength range to produce RTP. In particular, P2-M3, P2-M6, P2-M7 and P2-M9 films can be conveniently irradiated with a $365 \mathrm{~nm}$ portable UV lamp $\left(150 \mu \mathrm{W} \mathrm{cm} \mathrm{cm}^{-2}\right)$ to produce RTP. To further understand the relationship between chromophore molecular structures and 

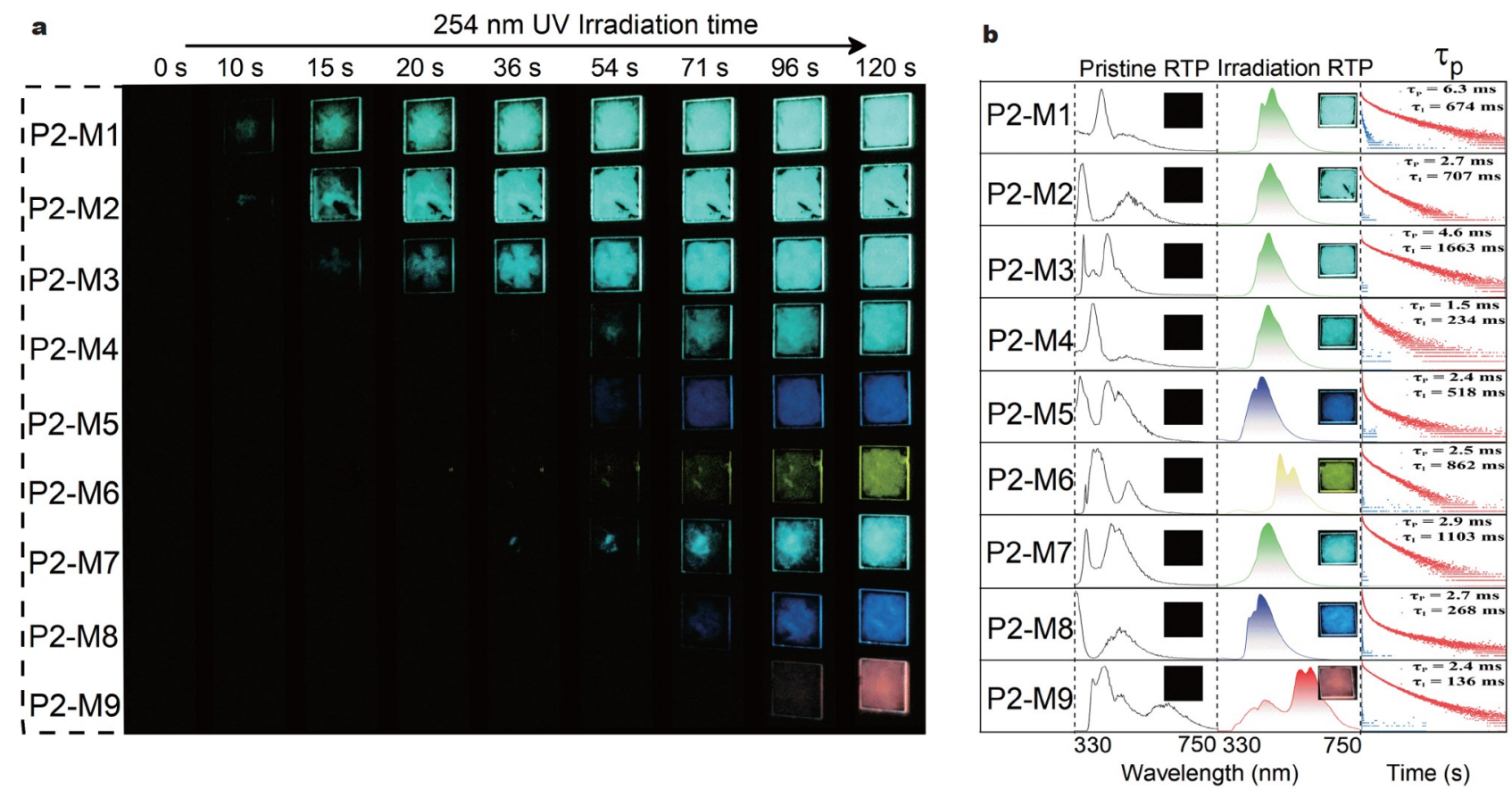

Figure 3 Images of P2-based films during the irradiation and their photophysical properties before and after the irradiation. (a) Pictures of the RTP phenomenon from different films after the UV lamp was turned off for $0.5 \mathrm{~s}$ at different irradiation times. (b) Phosphorescence spectra and phosphorescence lifetime spectra of these films before and after the irradiation. The insets are the images of the films (left) before and (right) after the irradiation.

dynamic stimulus-responsive RTP, hexa-(4-carboxyl-phenoxy)cyclotriphosphazene (donated as $\mathrm{G}$ ) synthesized in our previous work [43] was doped into P2. Compared with the nine kinds of chromophore molecules, the structure of $G$ is larger, and it contains a flexible bonds. Even when the triplet oxygen is consumed, it still has strong nonradiative transition ability. Thus, no RTP was observed from the UV-irradiated P2-G film. The phosphorescence intensity of the P2-G film after the irradiation was not obviously changed as compared with the pristine state (Fig. S27). These results demonstrate that small planar molecules are more suitable for this polymer encapsulation strategy, where the interactions between the small molecules and polymer matrix regulate the performance of RTP.

A series of copolymers (P1 and P3-P7) with different feeding ratios of VAc and AA as well as their individual homopolymers (PVAc and PAA) were synthesized to illustrate the role of polymer matrix in this strategy (Fig. S1). Then, M2 was doped into these different polymer matrices, defined as PVAc-M2, P1M2, P3-M2, P4-M2, P5-M2, P6-M2, P7-M2, PAA-M2, respectively. Similar to the P2-M2 film, no RTP was observed after rapidly exciting these films in the pristine state. After the irradiation with $254 \mathrm{~nm}$ UV light for a period of time, all films show RTP (Fig. S28 and Movie S3). When increasing the AA component in the polymer, the required irradiation time to trigger RTP becomes shorter. The photophysical properties before and after the irradiation are shown in Figs S29 and S30 as well as Table S3. Since the carboxylic acid group can form strong hydrogen bonding with the chromophore molecules, the phosphorescence lifetime of other films is longer than that of PVAcM2 film. Because the doping concentration of chromophore molecules in the film is very low $\left(0.1 \mathrm{mg} \mathrm{mL}^{-1}\right)$, the hydrogen bonding should be sufficient even when the content of the AA component in the polymer is low. Therefore, when the content of the AA component increases, the RTP properties of the resulting systems after irradiation would not show an obvious difference. Differential scanning calorimetry analysis was performed for all polymer matrices (Fig. S31). As the content of the AA component increases, the glass transition temperature $\left(T_{\mathrm{g}}\right)$ of the polymers generally exhibits an increasing trend. The increase of $T_{\mathrm{g}}$ indicates that the structure of the polymer becomes more rigid.

Four common commercially available polymers were selected as the matrix to illustrate the impact of polymer types on this strategy. They are polyacrylonitrile (PAN), polyvinylpyrrolidone (PVP), polyvinyl chloride (PVC) and polystyrene (PS). The chromophore M2 was doped into these four polymer matrices to form films, which were named PAN-M2, PVP-M2, PVC-M2, PS-M2, respectively. Expectedly, no RTP was detected in these films in their pristine states after being quickly excited by the UV lamp. After the UV irradiation for a longer period of time, RTP from the PVP-M2 and PAN-M2 films was observed, while the PVC-M2 and PS-M2 films still showed no RTP phenomenon (Fig. S32). The phosphorescence intensity of the PVC-M2 film after the irradiation exhibites a slight increase (Fig. S33), and the phosphorescence lifetime is only $1.5 \mathrm{~ms}$. The phosphorescence intensity of the PS-M2 film does not show an obvious change after the irradiation (Fig. S34). These results indicate that these two films do not have stimulus-responsive RTP behavior. The PMMA-M2 film was also prepared by the same method. As there is a large amount of $-\mathrm{COOCH}_{3}$ groups in the polymer chain, PMMA cannot form a strong hydrogen bonding network with the doped chromophore molecules. Thus, the irradiation time required for PMMA-M2 to produce RTP is longer than that of P2-M2 (Fig. S35), further proving that the hydrogen bonding 
is very important for the UV response speed of such irradiationdependent RTP systems. In addition, the RTP performance of P2-M2 after the irradiation is also better than that of PMMA-M2 (Fig. S36). The photophysical properties of chromophore M2 without any polymer matrix were conducted for comparison. Chromophore M2 did not exhibit the stimulus-responsive property, and its phosphorescent lifetime before and after irradiation was only $3 \mathrm{~ms}$ (Fig. S37). These results indicate that the polymer matrix plays a vital role in achieving long-lived RTP attributed to the strong intermolecular hydrogen bonding interactions formed between the polymer matrix and the chromophore molecule.

Then, the nonradiative transition rate $\left(K_{\mathrm{nr}}\right)[44,45]$ before and after the irradiation was calculated (Fig. 4a), since it is an important photophysical parameter in RTP. The $K_{\mathrm{nr}}$ of the P2M2 film before and after the irradiation is 366.74 and $1.29 \mathrm{~S}^{-1}$, respectively. Having such a low $K_{\mathrm{nr}}$ after the irradiation is one of the main reasons for its long lifetime. To further support our conclusion, the photophysical properties of the P2-M2 film were measured at low temperatures. At $77 \mathrm{~K}$, the film can produce obvious RTP, because low temperature can well suppress the nonradiative transition of the loaded chromophore molecules. As compared with the results at room temperature, the film has stronger RTP phenomenon (Fig. 4b) and longer phosphorescence lifetime (Fig. 4c) at low temperature, indicating that the dynamic stimulus-responsive RTP does not come from thermally activated delayed fluorescence [46]. The same conclusion can be drawn from its steady-state photoluminescence spectrum (Fig. S38) and fluorescence lifetime (Fig. S39).

The photophysical properties of P2-M2 in vacuum conditions were investigated to further demonstrate that this irradiation- dependent RTP results from the oxygen consumption mechanism. In vacuum conditions, $\mathrm{P} 2-\mathrm{M} 2$ can directly produce bright RTP, and there is no obvious improvement after the irradiation (Fig. S40). As shown in Fig. 4d, the phosphorescence intensity only slightly increases after the irradiation, and the phosphorescence lifetime does not show an obvious change (Fig. S41). Interestingly, the phosphorescence lifetime of P2-M2 in vacuum is longer than that irradiated for $5 \mathrm{~min}$ under ambient conditions. Even when P2-M2 is stored in vacuum for $12 \mathrm{~h}$, it can still produce bright RTP after being quickly excited by a UV lamp. All these results indicate that the irradiation-dependent RTP is mainly attributed to the oxygen consumption of the polymer matrix.

At the same time, the UV-vis absorption spectra of the films were also recorded. As shown in Fig. 4e, upon increasing the irradiation time, the absorption peaks of the P2-M2 film at 300 to $430 \mathrm{~nm}$ gradually increase, while the absorption intensity at $266 \mathrm{~nm}$ gradually decreases. After heating for $2 \mathrm{~min}$, the changes can be restored (Fig. S42). The UV-vis absorption spectra of other phosphor-doped P2 films show a similar phenomenon (Fig. S43). The PVP-M2, PAN-M2 and PVC-M2 films also exhibit similar experimental results (Fig. S44). In addition, electron paramagnetic resonance (EPR) spectrum [47] of the irradiated film shows three sets of signals, indicating the production of singlet oxygen after the irradiation (Fig. 4f).

Based on the unique dynamic stimulus-responsive RTP character, these materials were applied for the information storage. Three different patterns (flower, letters "CQUT" and numbers "888") were prepared using methanol solutions of P2M2 and P2-M6 as the inks. Different information can be observed under different wavelengths of UV light and different
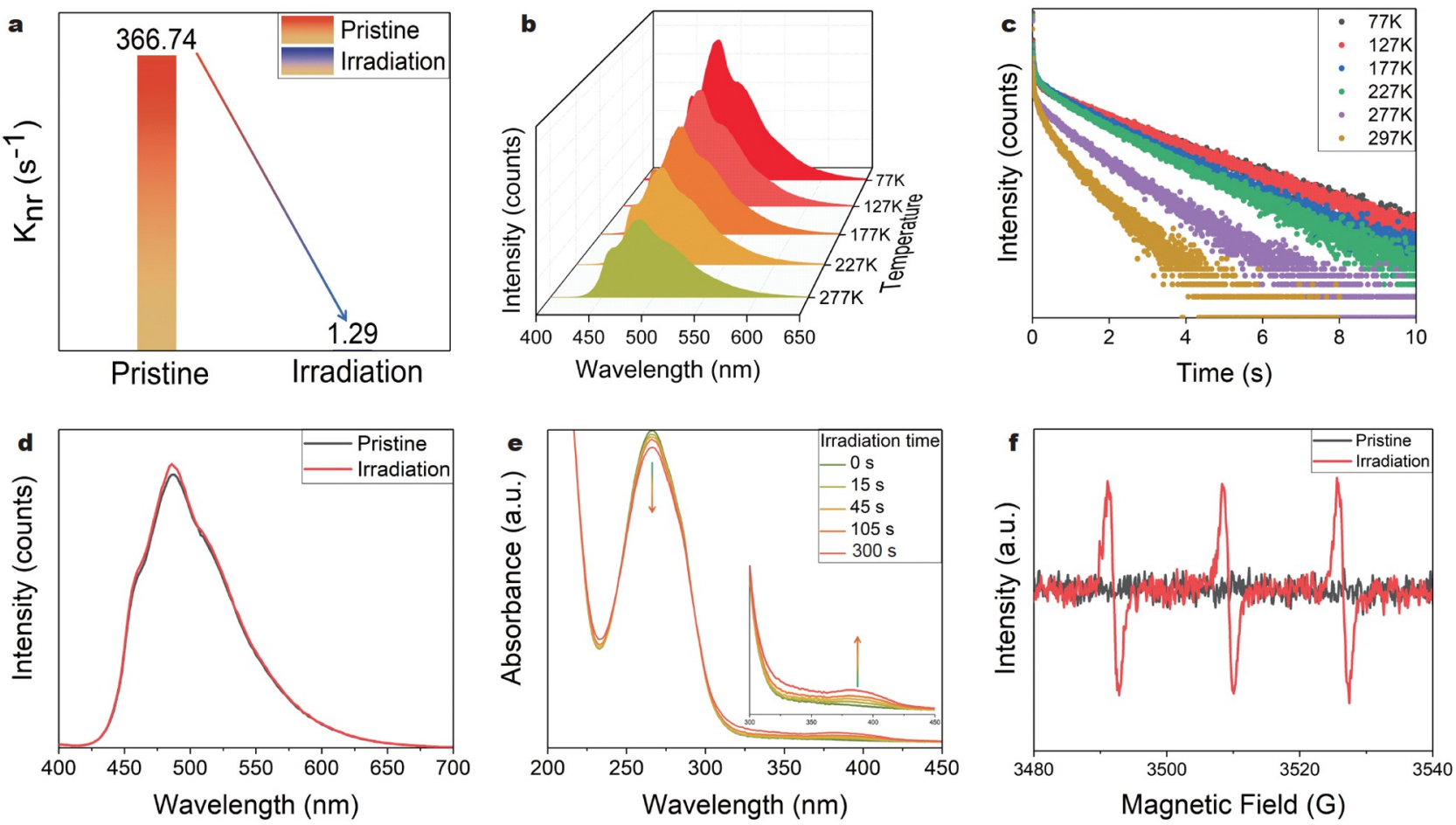

Figure 4 Mechanism of dynamic stimulus-responsive RTP systems. (a) $K_{\mathrm{nr}}$ values of the P2-M2 film before and after the irradiation. (b) Variable temperature-dependent phosphorescence spectra of the P2-M2 film. (c) Variable temperature-dependent phosphorescence lifetime of the P2-M2 film. (d) Phosphorescence spectra of the P2-M2 film before and after the irradiation in vacuum condition. (e) UV-vis absorption spectra of the P2-M2 film under different irradiation times. (f) EPR spectra of P2-M2 before and after the irradiation. 


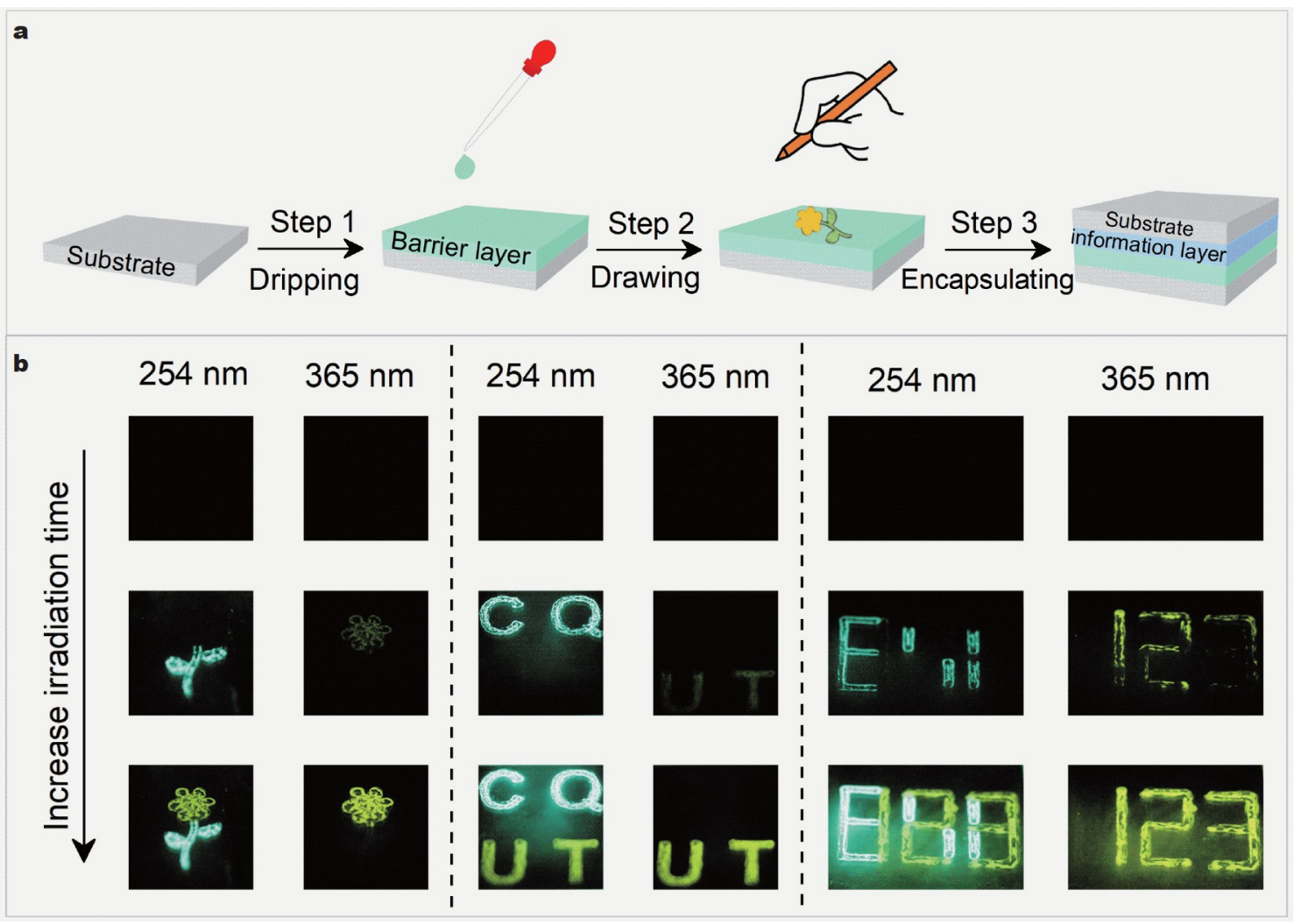

Figure 5 Demonstration of dynamic stimulus-responsive RTP systems for data storage under ambient conditions. (a) Pattern preparation procedure for the information storage. First, the barrier layer was coated on the substrate (e.g., glass, quartz, and plastic). Next, the methanol solutions of P2-M2 and P2-M6 were used as inks to draw the designed patterns on the barrier layer. Finally, after the patterns were dried, a barrier layer on the pattern was added. (b) RTP photographs of flower, "CQUT" and "888" after different irradiation times. The blue afterglow part of each pattern was prepared by P2-M2 ink, and the yellow afterglow part was prepared by P2-M6 ink. The RTP images were recorded after turning off a 254 or $365 \mathrm{~nm}$ hand-held UV lamp for $0.5 \mathrm{~s}$.

irradiation times (Fig. 5 and Fig. S45), thus achieving the information storage and encryption. At first, after rapid excitation with $254 \mathrm{~nm}$ UV light, no information can be seen. As the irradiation time increases, partial patterns appear. For example, only the leaf part can be observed in the flower pattern, and "CQUT" only shows two letters of "CQ", and the numbers " 888 " present a blurry pattern. As the irradiation time further increases, the complete patterns can be observed. When excited with $365 \mathrm{~nm}$ UV light instead, no information can be seen at first. Upon increasing the irradiation time, the petals, the letters "UT" and the numbers " 123 " can be observed. However, no matter how the irradiation time increases, only this part of the patterns is observed, because the other part of the patterns is made of the P2-M2 ink, and it cannot produce RTP after being irradiated by $365 \mathrm{~nm}$ UV light.

\section{CONCLUSION}

In summary, we have developed a strategy for realizing dynamic stimulus-responsive long-lived RTP systems through doping some common organic chromophores into polymer matrices prepared from VAC and AA with different feeding ratios. After UV light irradiation, the increasing process of the phosphorescence can be observed by the naked eye, and the photophysical properties of the systems can be improved by hundreds of times. Importantly, this strategy is general and universal, and many types of small chromophore molecules and polymer matrices are suitable for generating strong phosphorescence upon UV light irradiation. Based on the unique photophysical properties, these materials might be employed in the fields of information storage and encryption. Different information can be observed by controlling the wavelength of UV light and the time of irradiation. This strategy not only paves a way to construct simple, efficient, and commercially viable long-lived RTP polymer systems, but also expands the application scope of stimulus-responsive materials.

Received 1 July 2021; accepted 9 August 2021; published online 10 September 2021

1 Gu M, Shi H, Ling K, et al. Polymorphism-dependent dynamic ultralong organic phosphorescence. Research, 2020, 2020: 8183450

2 Su Y, Zhang Y, Wang Z, et al. Excitation-dependent long-life luminescent polymeric systems under ambient conditions. Angew Chem Int Ed, 2020, 59: 9967-9971

3 Zhang X, Du L, Zhao W, et al. Ultralong UV/mechano-excited room temperature phosphorescence from purely organic cluster excitons. Nat Commun, 2019, 10: 5161

4 Liu S, Ma Y, Liu S, et al. Achieving multiple emission states and controllable response behaviour in thermochromic luminescent materials for security applications. J Mater Chem C, 2020, 8: 10798-10804

5 Wang Y, Yang J, Fang M, et al. Förster resonance energy transfer: An efficient way to develop stimulus-responsive room-temperature phosphorescence materials and their applications. Matter, 2020, 3: 449-463

6 Kim DH, D’Aléo A, Chen XK, et al. High-efficiency electroluminescence and amplified spontaneous emission from a thermally activated delayed fluorescent near-infrared emitter. Nat Photon, 2018, 12: $98-104$ 
7 Ogoshi $\mathrm{T}$, Tsuchida $\mathrm{H}$, Kakuta $\mathrm{T}$, et al. Ultralong room-temperature phosphorescence from amorphous polymer poly(styrene sulfonic acid) in air in the dry solid state. Adv Funct Mater, 2018, 28: 1707369

8 He Z, Gao H, Zhang S, et al. Achieving persistent, efficient, and robust room-temperature phosphorescence from pure organics for versatile applications. Adv Mater, 2019, 31: 1807222

9 Gu L, Shi H, Bian L, et al. Colour-tunable ultra-long organic phosphorescence of a single-component molecular crystal. Nat Photonics, 2019, 13: 406-411

10 Genovese D, Aliprandi A, Prasetyanto EA, et al. Mechano- and photochromism from bulk to nanoscale: Data storage on individual selfassembled ribbons. Adv Funct Mater, 2016, 26: 5271-5278

11 Zhang T, Wang C, Ma X. Metal-free room-temperature phosphorescent systems for pure white-light emission and latent fingerprint visualization. Ind Eng Chem Res, 2019, 58: 7778-7785

12 Zhao W, He Z, Lam JWY, et al. Rational molecular design for achieving persistent and efficient pure organic room-temperature phosphorescence. Chem, 2016, 1: 592-602

13 He Z, Zhao W, Lam JWY, et al. White light emission from a single organic molecule with dual phosphorescence at room temperature. Nat Commun, 2017, 8: 416

14 Xiao L, Wu Y, Yu Z, et al. Room-temperature phosphorescence in pure organic materials: Halogen bonding switching effects. Chem Eur J, 2018, 24: 1801-1805

15 Bolton O, Lee K, Kim HJ, et al. Activating efficient phosphorescence from purely organic materials by crystal design. Nat Chem, 2011, 3: 205-210

16 Shoji Y, Ikabata Y, Wang Q, et al. Unveiling a new aspect of simple arylboronic esters: Long-lived room-temperature phosphorescence from heavy-atom-free molecules. J Am Chem Soc, 2017, 139: 27282733

17 Yang X, Yan D. Strongly enhanced long-lived persistent room temperature phosphorescence based on the formation of metal-organic hybrids. Adv Opt Mater, 2016, 4: 897-905

18 Kuila S, Garain S, Bandi S, et al. All-organic, temporally pure white afterglow in amorphous films using complementary blue and greenishyellow ultralong room temperature phosphors. Adv Funct Mater, 2020, 30: 2003693

19 Cai S, Ma H, Shi H, et al. Enabling long-lived organic room temperature phosphorescence in polymers by subunit interlocking. Nat Commun, 2019, 10: 4247

$20 \mathrm{Wu} \mathrm{H}$, Chi W, Chen $\mathrm{Z}$, et al. Achieving amorphous ultralong room temperature phosphorescence by coassembling planar small organic molecules with polyvinyl alcohol. Adv Funct Mater, 2019, 29: 1807243

21 Yao X, Wang J, Jiao D, et al. Room-temperature phosphorescence enabled through nacre-mimetic nanocomposite design. Adv Mater, 2021, 33: 2005973

22 Ding B, Ma L, Huang Z, et al. Engendering persistent organic room temperature phosphorescence by trace ingredient incorporation. Sci Adv, 2021, 7: eabf9668

23 Zhang $\mathrm{T}$, Ma X, Wu $\mathrm{H}$, et al. Molecular engineering for metal-free amorphous materials with room-temperature phosphorescence. Angew Chem Int Ed, 2020, 59: 11206-11216

24 Zhao C, Jin Y, Wang J, et al. Heavy-atom-free amorphous materials with facile preparation and efficient room-temperature phosphorescence emission. Chem Commun, 2019, 55: 5355-5358

25 Ma X, Wang J, Tian H. Assembling-induced emission: an efficient approach for amorphous metal-free organic emitting materials with room-temperature phosphorescence. Acc Chem Res, 2019, 52: 738-748

$26 \mathrm{Gu} \mathrm{L}, \mathrm{Wu} \mathrm{H}, \mathrm{Ma} \mathrm{H}$, et al. Color-tunable ultralong organic room temperature phosphorescence from a multicomponent copolymer. Nat Commun, 2020, 11: 944

27 Lin X, Wang J, Ding B, et al. Tunable-emission amorphous roomtemperature phosphorescent polymers based on thermoreversible dynamic covalent bonds. Angew Chem Int Ed, 2021, 60: 3459-3463

28 Zhang T, Wu Y, Ma X. Tunable multicolor room-temperature phosphorescence including white-light emission from amorphous copolymers. Chem Eng J, 2021, 412: 128689

29 Gu L, Wang X, Singh M, et al. Organic room-temperature phosphor- escent materials: from static to dynamic. J Phys Chem Lett, 2020, 11: 6191-6200

30 Yang J, Fang M, Li Z. Stimulus-responsive room temperature phosphorescence in purely organic luminogens. InfoMat, 2020, 2: 791-806

31 Li Q, Li Z. Molecular packing: another key point for the performance of organic and polymeric optoelectronic materials. Acc Chem Res, 2020, 53: 962-973

32 Li Q, Li Z. Miracles of molecular uniting. Sci China Mater, 2020, 63: 177-184

33 Wang Y, Yang J, Gong Y, et al. Host-guest materials with room temperature phosphorescence: Tunable emission color and thermal printing patterns. SmartMat, 2020, 1: e1006

34 Dang Q, Hu L, Wang J, et al. Multiple luminescence responses towards mechanical stimulus and photo-induction: The key role of the stuck packing mode and tunable intermolecular interactions. Chem Eur J, 2019, 25: 7031-7037

35 Yang J, Zhen X, Wang B, et al. The influence of the molecular packing on the room temperature phosphorescence of purely organic luminogens. Nat Commun, 2018, 9: 840

36 Kanosue K, Ando S. Polyimides with heavy halogens exhibiting roomtemperature phosphorescence with very large Stokes shifts. ACS Macro Lett, 2016, 5: 1301-1305

37 DeRosa M. Photosensitized singlet oxygen and its applications. Coord Chem Rev, 2002, 233-234: 351-371

38 Ma L, Sun S, Ding B, et al. Highly efficient room-temperature phosphorescence based on single-benzene structure molecules and photoactivated luminescence with afterglow. Adv Funct Mater, 2021, 31: 2010659

39 Gmelch M, Thomas H, Fries F, et al. Programmable transparent organic luminescent tags. Sci Adv, 2019, 5: eaau7310

40 Louis $\mathrm{M}$, Thomas $\mathrm{H}$, Gmelch $\mathrm{M}$, et al. Blue-light-absorbing thin films showing ultralong room-temperature phosphorescence. Adv Mater, 2019, 31: 1807887

41 Zang L, Shao W, Kwon MS, et al. Photoresponsive luminescence switching of metal-free organic phosphors doped polymer matrices. Adv Opt Mater, 2020, 8: 2000654

42 Wang Z, Zhang Y, Wang C, et al. Color-tunable polymeric long-persistent luminescence based on polyphosphazenes. Adv Mater, 2020, 32: 1907355

43 Su Y, Phua SZF, Li Y, et al. Ultralong room temperature phosphorescence from amorphous organic materials toward confidential information encryption and decryption. Sci Adv, 2018, 4: eaas9732

44 Bhattacharjee I, Hirata S. Highly efficient persistent room-temperature phosphorescence from heavy atom-free molecules triggered by hidden long phosphorescent antenna. Adv Mater, 2020, 32: 2001348

45 Feng HT, Zeng J, Yin PA, et al. Tuning molecular emission of organic emitters from fluorescence to phosphorescence through push-pull electronic effects. Nat Commun, 2020, 11: 2617

46 Chen XK, Kim D, Brédas JL. Thermally activated delayed fluorescence (TADF) path toward efficient electroluminescence in purely organic materials: molecular level insight. Acc Chem Res, 2018, 51: 2215-2224

47 Gao R, Mei X, Yan D, et al. Nano-photosensitizer based on layered double hydroxide and isophthalic acid for singlet oxygenation and photodynamic therapy. Nat Commun, 2018, 9: 2798

Acknowledgements This work was financially supported by the National Natural Science Foundation of China (21875025), the Special Program of Chongqing Science and Technology Commission (cstc2018jcyjAX0296), the Innovation Research Group at the Institutions of Higher Education in Chongqing (CXQT19027), the Science and Technology Research Program of Chongqing Municipal Education Commission (KJZD-K201801101), Chongqing Talent Program, the Science and Technology Project of Banan District, and the Innovation Support Plan for the Returned Overseas of Chongqing (cx2020052). The research was also supported by Singapore Academic Research Fund (RT12/19 and MOE-MOET2EP10120-0003).

Author contributions Wang $\mathrm{Z}$ and Yang $\mathrm{C}$ conceived the idea; Wang $\mathrm{Z}$, Zheng $\mathrm{Y}$, and $\mathrm{Su} \mathrm{Y}$ carried out the synthesis, characterization, and analysis; 
Gao L, Zhu, Y, Xia J, Zhang Y, Wang C, Zheng X, and Li Y contributed to the measurements and data analyses; Wang $\mathrm{Z}$ and Yang $\mathrm{C}$ wrote the manuscript; Zhao $\mathrm{Y}$ and Yang $\mathrm{C}$ supervised the project. All authors discussed and reviewed the final version.

Conflict of interest The authors declare no conflict of interest.

Supplementary information Experimental details and supporting data are available in the online version of the paper.

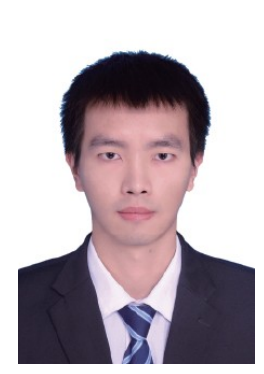

Zhonghao Wang is now a Master candidate at the School of Materials Science and Engineering, Chongqing University of Technology. His research interest focuses on the organic long-lived roomtemperature phosphorescence.

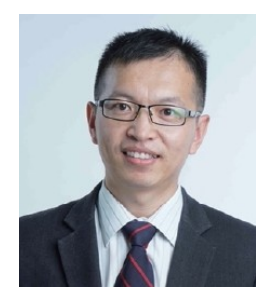

Yanli Zhao is currently the Lee Soo Ying Professor at Nanyang Technological University, Singapore. He received his $\mathrm{BSc}$ and $\mathrm{PhD}$ degrees under the supervision of Prof. Yu Liu from Nankai University. He was a postdoctoral scholar with Prof. Sir Fraser Stoddart (University of California, Los Angeles and subsequently Northwestern University) and Prof. Jeffrey Zink (University of California, Los Angeles). His current research focuses on integrated nanosystems for disease diagnostics and therapeutics, as well as porous nanomaterials for energy storage and catalysis.

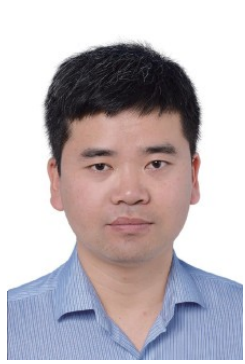

Chaolong Yang received his $\mathrm{PhD}$ degree in polymer chemistry and physics from the Chinese Academy of Sciences in 2013. Then, he joined the School of Materials Science and Engineering, Chongqing University of Technology as an assistant professor. During September 2016 to October 2017, he worked at Nanyang Technological University, Singapore as a Research Fellow, where he focused on the design and development of organic optoelectronic materials. $\mathrm{He}$ has been a full professor at Chongqing University of Technology since November 2018. His research concerns polymers and optoelectronic materials and their applications.

\section{紫外光激活有机小分子掺杂聚合物体系的长寿命室 温磷光}

王中豪 ${ }^{1 \dagger}$, 郑言 ${ }^{1 \dagger}$, 苏艳 ${ }^{1 \dagger}$, 高亮 ${ }^{1}$, 朱银银 ${ }^{1}$, 夏杰 $^{1}$, 张永锋 ${ }^{1}$, 王畅 ${ }^{1}$, 郑贤 ${ }^{1}$, 赵彦利 ${ }^{2^{*}}$, 杨朝龙 ${ }^{1,2^{*}}$, 李又兵 ${ }^{1}$

摘要 长寿命有机室温磷光(RTP)材料因其优异的性能和应用前景而 备受关注. 目前新型RTP材料的开发主要是通过合成新的发色团分子, 然后再通过结晶工程来实现. 然而, 新发色团分子的合成和结晶材料的 制备都存在巨大挑战. 在本工作中, 我们利用醋酸乙烯酯和丙烯酸的共 聚物作为基质, 将有机发色团分子掺杂到基质中, 制备了一系列余辉颜 色不同的动态刺激响应长寿命 RTP材料. 通过紫外光辐照, 可以在 10 秒 内观察到RTP从无到有的增强过程. 以P2-M2薄膜为例, 辐照后其磷光 强度、寿命、余辉亮度和量子产率分别提升了155、262、414和8倍. 这种独特的光物理现象归因于聚合物基质在紫外光照射下的耗氧特 性. 同时, 我们探索了这些刺激响应RTP材料在信息存储领域的潜在应 用. 本工作为制备简便、成本低和普适性高的小分子掺杂聚合物 RTP 体系提供了一种新策略. 